\title{
Lipid and exocrine pancreatic ultrastructural changes due to experimental diabetes
}

\author{
R. Maciejewski ${ }^{1}$, K. Burski ${ }^{1}$, F. Burdan ${ }^{1}$, Barbara Madej ${ }^{1}$, \\ M. Kopieniak ${ }^{2}$
}

\author{
${ }^{1}$ Department of Human Anatomy, Medical University of Lublin, Lublin, Poland \\ ${ }^{2}$ Department of Anaesthesiology, Clinical Hospital No 4, Lublin, Poland
}

Received: January 3, 2001

Accepted: April 30, 2001

\begin{abstract}
The aim of this study was to establish if the changes in the ultrastructure of the exocrine part of the pancreas are correlated with changes in serum glucose, cholesterol and lipoprotein fractions during the progression of diabetes in rabbits. Diabetes mellitus was induced in male New Zealand rabbits by a single injection of alloxan into the auricular vein. On the day 7 th the glucose level in the whole blood was measured and this day was designated as the first day of diabetes. Rabbits were divided into 5 groups: untreated control, 21-day diabetes, 42-day diabetes, 90-day diabetes and 180-day diabetes. The cholesterol, HDL (high-density lipoprotein) and LDL (low-density lipoprotein) levels were examined in the serum. The total pancreatic lipase activity was measured spectrophotometrically in the pancreatic homogenate. Histological specimens were examined under an electron microscopy. The glucose level increased significantly in all of the alloxan exposed animals. The significant elevation of cholesterol level was observed on day 21 and 180. The HDL level was increased $(\mathrm{P}<0.05)$ only on the day 21 st. The LDL level and the total activity of pancreatic lysosomal lipase increased significantly on day 21, 42 and 90 . Further dilation of granular endoplasmic reticular ducts and decrease in the number of zymogen granules were observed amongst exocrine cells. Fragmented mitochondrial and translucent matrix were also seen. Intensification of the pancreatic fibrosis was found on day 90. Microvascular changes were reported in exocrine cells after 180 days. Their nuclei were smaller with large bulges on the nuclear membrane, and the number of heterogeneous electron granules of zymogen further declined. We concluded that the intensification of ultrastructural changes of the exocrine part of the pancreas correlated with the changes of the pancreatic lipase activity, and glucose and lipoprotein levels.
\end{abstract}

Correspondence should be addressed to

Ryszard Maciejewski

Department of Human Anatomy

Medical University of Lublin

20-074 Lublin, 1 Spokojna Str., Poland

Phone: +48-81-5322118

Fax: +48-81-5328903 
Keywords: lipid disorders, lipase activity, pancreas, diabetes mellitus, serum glucose, cholesterol, lipoprotein fractions, rabbit, HDL, LDL, pancreatic fibrosis

Numerous publications in recent years stress the importance of exocrine pancreatic functions in the course of diabetes mellitus $(3,6,7,20,22)$. This was observed mostly in patients with insulin-dependent diabetes. It is known that due to insulin deficiency and relative glucagon predominance, the patients suffer from exocrine cells degranulation and atrophy $(6,7,22)$. Clinical investigations and laboratory research supported with morphological and ultrastructural observations, after having proven the close relation between exo- and endocrine parts, lead to quantitative evaluation of biochemical and morphological disorders related to diabetes mellitus (20).

Various articles regarding influence of insulin deficiency on pancreatic exocrine function describe distinctive changes in composition and quality characteristics of pancreatic juice (2). It is particularly interesting that the relation between insulin and glucagon concentrations and their antagonistic influence on exocrine functions seem to play the key role in these disorders. Insulin is recognized as a main factor stimulating exocrine activity, whilst the inhibiting action is ascribed to glucagon $(7,13)$.

The impairment of pancreatic exocrine functions was proven in clinical investigations in diabetic patients. It was reflected both in functional tests as a decrease of enzymatic activity and ionic shifts, and in histopathological observations in the form of involution changes starting from decrease in number of granules with exocrine cell atrophy at the end $(6,7,22)$.

The aim of this study was to establish if the changes in the ultrastructure of the exocrine part of the pancreas are correlated with changes in serum glucose, cholesterol and lipoprotein levels, and activity of the pancreatic lysosomal lipase during the progression of diabetes in rabbits.

\section{Materials and Methods}

Eighty-nine male rabbits, New Zealand breed (Experimental Animals Laboratory, Chorzelow, Warsaw, Poland), weighing $2.750-3.300 \mathrm{~kg}$ were used in the experiment. Animals were housed one per cage under $12 \mathrm{~h} / 12 \mathrm{~h}$ light/dark cycle at $21 \pm 2{ }^{\circ} \mathrm{C}$ temperature and $50 \%$ relative humidity with standard granulated food and water available ad libitum.

Bioethical Committee of Medical University of Lublin approved the experimental protocol.

After two weeks of acclimatisation the animals were randomly divided into untreated control (Group 1, n=18) and diabetic groups (Groups 2-5). Diabetes mellitus was induced by a single injection of alloxan (Sigma Chemical Company; St. Louis, MO, 
USA) at a dose of $10 \mathrm{mg} / \mathrm{kg}$ into the auricular vein (15). On day 7 the glucose level in the whole blood was measured by a lactometer (Boehringer, Germany) to confirm the presence of diabetes. This day was considered as the start of the decrease. The animals were divided into the following diabetic groups: Group $2-21$ days diabetes mellitus $(\mathrm{n}=18)$, Group $3-42$ days diabetes mellitus $(\mathrm{n}=17)$, Group $4-90$ days diabetes mellitus $(\mathrm{n}=19)$, Group 5 -180 days diabetes mellitus $(\mathrm{n}=17)$. After the above-mentioned periods blood samples were taken and the rabbits were anaesthetized and sacrificed by decapitation. The final level of glucose in the sera was determined spectrophotometrically by enzymatic method using ready kit GS-120L (Cormay, Lublin, Poland) at wavelength of $500 \mathrm{~nm}$.

The pancreas was removed and stored at $-20^{\circ} \mathrm{C}$. The samples were defrosted in $0.9 \%$ solution of $\mathrm{NaCl}$ at $4{ }^{\circ} \mathrm{C} .1 \mathrm{~g}$ of the tissue of each sample was taken for biochemical investigation. They were placed into a $0.3 \mathrm{M}$ sucrose solution at $4{ }^{\circ} \mathrm{C}$ in proportions of $1 \mathrm{~g}$ of tissue to $5.0 \mathrm{ml}$ of sucrose and homogenized. The obtained homogenate was centrifuged for 10 minutes at $2.200 \mathrm{~g}$ at $4{ }^{\circ} \mathrm{C}$. The supernatant was decanted and centrifuged for 20 minutes at $35.000 \mathrm{~g}$. The obtained sample containing the free fraction of enzyme was assigned as supernatant 1 (19). The precipitate was placed into $5.0 \mathrm{ml}$ of $0.3 \mathrm{M}$ sucrose containing $0.1 \%$ Triton $\mathrm{X}-100$ and stored for 24 hours at $4{ }^{\circ} \mathrm{C}$. Then the precipitate was centrifuged for 20 minutes at $35,000 \mathrm{~g}$. The supernatant, containing the fraction of bound enzyme, was decanted and assigned as supernatant 2. The sum of the free and bound fraction presented the total activity of pancreatic lysosomal lipase. Lipase (EC 3.1.1.3) activity assay was based on the fact that the enzymes examined decompose a specific substrate releasing free 4metyloumbeliferol (4). Spectrophotometric evaluation was performed using substrates (Sigma), which form colored complexes reacting with the proteases (16). Lipase activity was expressed in nmol/1mg of protein/1 hour of incubation.

The principle of the method of cholesterol measurement was based on oxidation of free cholesterol to cholestenon releasing hydrogen peroxide, which in the presence of 4-aminophenason and phenol is converted quantitatively to chromogen. For this measurement Boehringer Mannheim (Vienna, Austria) set was used in that measurement (23).

Determination of lipoprotein fractions was based on indirect methods consisting of the precipitation of specific lipoprotein fractions; and of the enzymatic measurement, of cholesterol included in the supernatant of the given fraction (12).

HDL (high-density lipoprotein) cholesterol was determined in the supernatant after LDL (low-density lipoprotein) and VLDL (very low-density lipoprotein) had been precipitated using magnesium ions and phosphotungstic acid. LDL cholesterol concentration was determined after its selective precipitation using polyvinyl sulphate. LDL cholesterol was a difference between the total cholesterol concentration and 
concentration in supernatant which remained after the sediment was removed by centrifugation (2). Extinction was measured on spectrophotometer SPM 90A at wavelength $546 \mathrm{~nm}$. Concentration was calculated by multiplying results by the coefficient of extinction. Results are presented in $\mathrm{mmol} / \mathrm{l}$.

For ultrastructure examination the pancreas was obtained from randomly picked three animals in each group, was removed and fixed. Fixation procedure was done according to standard methods $(8,10)$. The samples were fixed for 4 hours in $4 \%$ glutar aldehyde, in $0.1 \mathrm{M}$ cacodylate buffer of $\mathrm{pH} 7.4$, at $+4{ }^{\circ} \mathrm{C}$. The specimens were then washed twice for 10 minutes with 0.1 cacodylate buffer and prefixed for 2 hours in $1 \%$ osmium tetroxide in the same buffer at a temperature $+4{ }^{\circ} \mathrm{C}$. After dehydration in a series of ethanol solution and propylene oxide, the specimens were saturated with SPURR Low-Viscosity Polysciences resin. Ultra-thin specimens were prepared by use of Richer Om-U3 ultramicrotome, and subsequently observed and documented by Tesla BS-500 microscope.

The statistical analysis was done using the SAS system v. 6.11 (SAS Institute Inc., SAS Campus Drive, Carry, NC 27513, USA). Results are expressed as Means \pm SD (standard deviation). Differences between groups were analyzed by one-way ANOVA. The correlation coefficients $(r)$ between analyzed characters $(X, Y)$ were counted. If $\mathrm{P}<0.05$, differences between the mean values were considered statistically significant.

\section{Results}

Statistical characterisation of biochemical parameters is presented in Table I.

Table I

The level of glucose, cholesterol, $H D L$ and $L D L(m m o l / l)$ and the total activity of pancreatic lysosomal lipase (nmol/1 mg of protein/1h) in control (Group 1) and diabetic groups (Groups 2-5)

\begin{tabular}{lccccc}
\hline \multicolumn{7}{c}{ Experimental groups } \\
\cline { 2 - 7 } & $\begin{array}{c}1 \\
\text { (control) }\end{array}$ & $\begin{array}{c}2 \\
(21 \text { days })\end{array}$ & $\begin{array}{c}3 \\
(42 \text { days })\end{array}$ & $\begin{array}{c}4 \\
(90 \text { days })\end{array}$ & $\begin{array}{c}5 \\
(180 \text { days })\end{array}$ \\
\hline $\begin{array}{l}\text { Number } \\
\text { of animals }\end{array}$ & 18 & 18 & 17 & 19 & 17 \\
Glucose & $6.36 \pm 2.03$ & $21.79 \pm 9.49^{*}$ & $32.02 \pm 19.1^{*}$ & $31.33 \pm 19.2^{*}$ & $23.15 \pm 10.8^{*}$ \\
Cholesterol & $1.35 \pm 0.18$ & $1.91 \pm 0.42^{*}$ & $1.62 \pm 0.27$ & $1.70 \pm 0.37$ & $2.19 \pm 0.53^{*}$ \\
HDL & $0.44 \pm 0.11$ & $0.61 \pm 0.15^{*}$ & $0.52 \pm 0.14$ & $0.51 \pm 0.17$ & $0.36 \pm 0.09$ \\
LDL & $0.41 \pm 0.13$ & $0.84 \pm 0.19^{*}$ & $0.86 \pm 0.23^{*}$ & $0.62 \pm 0.15^{*}$ & $0.56 \pm 0.22$ \\
Lipase & $71.48 \pm 14.6$ & $103 \pm 10.94^{*}$ & $224.6 \pm 38.5^{*}$ & $124.1 \pm 15.1^{*}$ & $90.31 \pm 10.2$ \\
\hline
\end{tabular}

$* \mathrm{P}<0.05$ as compared with control (Group 1) 


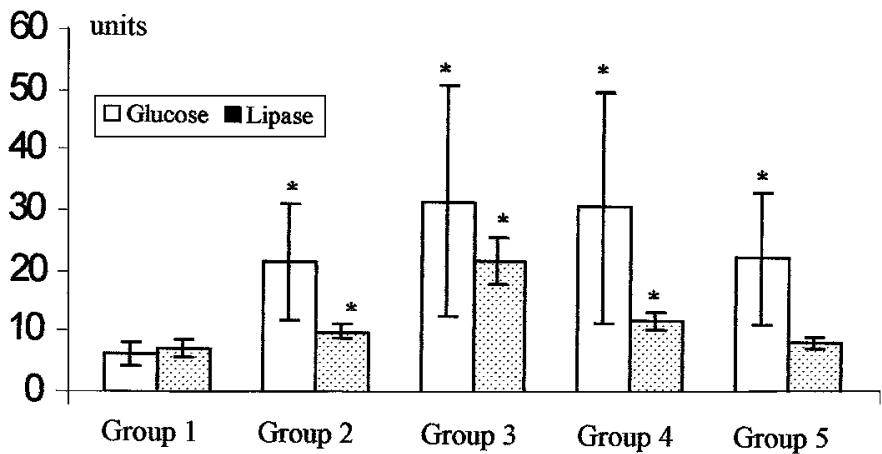

Fig. 1. Changes of serum glucose level $(\mathrm{mmol} / \mathrm{l})$ and total activity of pancreatic lysosomal lipase $\left(10^{-1} \mathrm{nmol} / \mathrm{mg} / \mathrm{h}\right)$ in control (Group 1) and diabetic groups (Groups 2-5)

The glucose level increased significantly in the all alloxan exposed animals. The significant elevation of cholesterol level was observed on day 21 and 180. The HDL level was increased $(\mathrm{P}<0.05)$ only on the day 21 st. The LDL level and total activity of lipase increased significantly on day 21,42 and 90 . The changes of the total pancreatic lysosomal lipase activity followed the changes of glucose in serum (Fig. 1).

Regular view of exocrine cells was observed in ultra thin preparations of rabbits' pancreas in the control group examined under the electron microscope. It was characterized by large oval nuclei, abundant granular endoplasmic reticulum in layers, and numerous zymogene granules of homogenous saturation, gathered around the lumen of pancreatic acini in the apical regions of the cells. Regular mitochondria with typical crest structures were observed in exocrine cells. Vascular walls and excretory ducts remained also unchanged.

Ultrastructural images of pancreas obtained from animals with 21 days of diabetes manifested certain differences in comparison with the control. Granular endoplasmic reticular ducts were dilated, which in the ultra thin preparation was described as the sign of microvacuolar degeneration. Decrease in the quantity of zymogene granules revealed in electron microscope was confirmed in the ultrastructural image. Vessels and excretory ducts showed thickening of the walls with fibrocytes' adhesion around them, which in the ultra thin preparations was described as an initial stage of pancreatic fibrosis. Concentration of glycogen around cell nuclei was a sign of its storage.

More significant changes of new quality were observed in ultra thin preparations of pancreas in rabbits with 42 days diabetes. Some of exocrine cells revealed the features of initial necrosis characterized by the presence of dead cytoplasm regions, as observed in electron microscope. Additional ducts in mitochondrial structure were noted in exocrine cells. Mitochondrial crests were fragmented and matrix became 
translucent. An appearance of myelin structures within the mitochondrial matrix was also stated, which can be described as a myelin degeneration of these cells.

Concentration of fibrocytes was noticed in the surroundings of thickened walls of exocrine ducts and vessels. Large vacuoles filled up with amorphic masses appeared in exocrine cells. Similar phenomenon was observed in the vascular endothelial cells (Fig. 2).

Further progress in ultrastructural changes was observed in the preparations of pancreas in animals with 90 days of diabetes. Pancreatic fibrosis intensification resulted in further dilation of extracellular space, with large quantities of collagen and microfibrillar protein substance were found. Previously reported dilation of rough endoplasmic reticular ducts and the presence of few zymogene granules of heterogeneous electron density in exocrine cells. Eosinophilic granulocytes appeared in the perivascular space. Further changes of myelin mitochondrial degeneration were noticed in exocrine cells, as well as larger glycogen accumulation in cytoplasm as the sign of its persisting storage process could be observed. These led to the general degradation of cytoplasm structures. Large vacuoles and lysosomes were also present (Fig. 3).

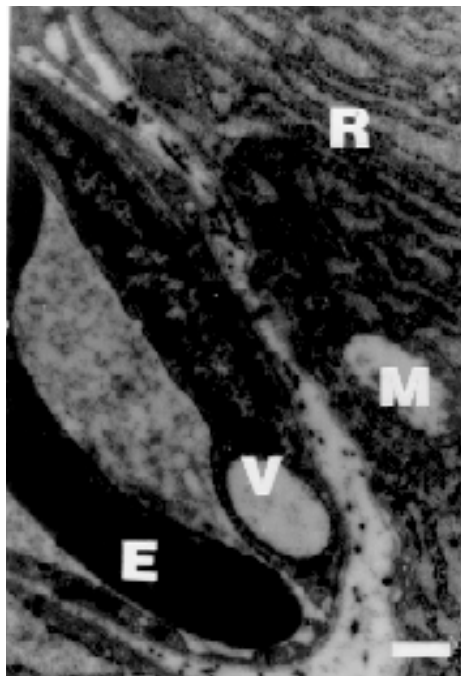

Fig. 2. Exocrine part of the pancreas in rabbit, 42-day diabetes. Vacuole inside the vascular endothelial cell (V) and dilated endoplasmic reticulum (R). Mitochondrion with fragmented crests and translucent matrix (M). Erythrocyte inside capillary (E). Calibration bar $-1.0 \mu \mathrm{m}$

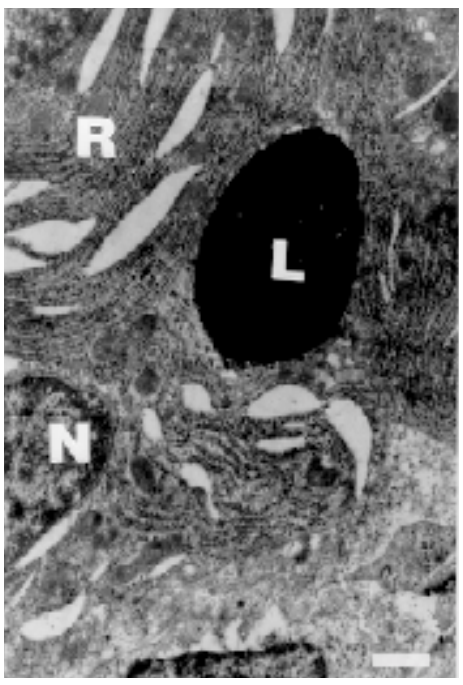

Fig. 3. Exocrine part of the pancreas in rabbit, 90-day diabetes. Widened and dilated reticulum $(\mathrm{R})$ and large lysosome (L) filled by dense mass. Glycogen around nucleus (N). Calibration bar $-2.5 \mu \mathrm{m}$ 
Advanced stage of disorders already described in former experimental groups was reported in ultra thin preparations of pancreas in rabbits with 180 days diabetes. Deep microvacuolar changes, that are dilation of rough endoplasmic reticular ducts and numerous large vacuoles with myelin figures, were reported in exocrine cells. Their nuclei were smaller with large bulges on the nuclear membrane, and the number of heterogeneous electron granules of zymogene declined further. Light granules containing immature products of cellular synthesis dominated. Pancreatic fibrosis intensified, which was manifested by the presence of the whole bundles of collagen and microfibrillar protein material in dilated extracellular and perivascular spaces and around thickened vascular and exocrine ducts' walls (Fig. 4 and Fig. 5).

\section{Discussion}

Systemic and metabolic changes observed in our study were accompanied by the corresponding transformations on pancreatic ultrastructural level. Number and intensity of these changes were proportional to the length of the alloxan-induced diabetes.

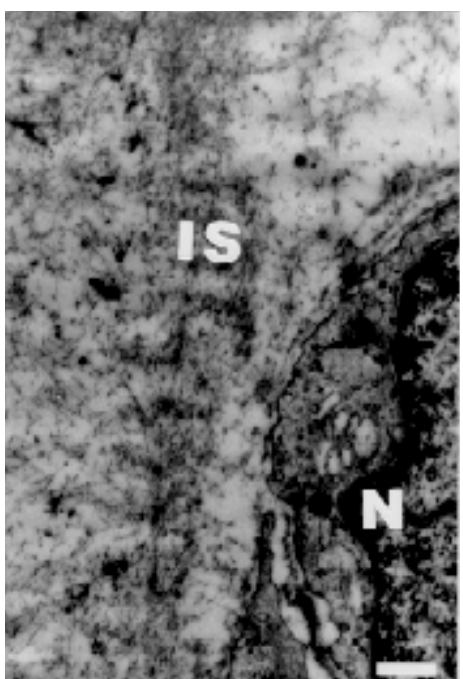

Fig. 4. Exocrine part of the pancreas in rabbit, 180-day diabetes. Considerably enlarged intercellular space (IS) filled by microfibrillar protein material. Cell nucleus $(\mathrm{N})$. Calibration bar $-1.25 \mu \mathrm{m}$

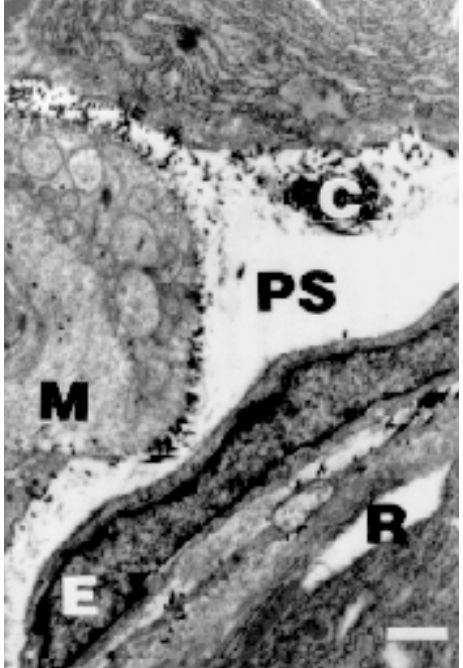

Fig. 5. Exocrine part of the pancreas in rabbit, 180-day diabetes. Massive edema of perivascular space (PS) with the presence of protein material (M) and the bundles of collagen (C). Endothelial cell (E) and dilated reticulum (R). Calibration bar $-2.5 \mu \mathrm{m}$ 
Examined samples collected from each of the experimental groups showed various abnormalities from the beginning of the observation that is from day 21. One of the earliest noted changes was the appearance of a large number of small vacuoles initially in the exocrine cells, which was seen as rough endoplasmic reticulum dilation.

This phenomenon had an increasing tendency in the 42 and 90 days diabetes. In pancreatic preparations of 180 days diabetes animals numerous, large vacuoles with enclosed myelin figures were observed. They manifested an advanced process of cellular structure degradation at this stage of the experiment. Above results are conformable with reports of other authors about the changes evoked in various parenchymal organs of animals with alloxan-induced diabetes $(1,3,14,17,18)$. Adecrease was revealed in total number of zymogene granules, besides microvacuolar degeneration in exocrine cells as early as on 21 day of disease. Heterogeneous density of these structures was observed in electron microscope, which supported the thesis of impairment of zymogene maturation in exocrine cells. This phenomenon was increasing parallel to diabetes development, and on day 180 granules of immature structure dominated over the number of regular zymogene. Total number of these structures was also significantly decreasing in the course of the experiment. These results are supported with other reports of decline in the quantity of peptide enzymes in pancreatic juice in the course of diabetes $(14,21)$. Above observations may be regarded as an ultrastructural confirmation of biochemical quantitative assay of products of pancreatic exocrine activity.

Dilation of extracellular space, which occurred already in 21 days samples, may be also recognized as an early stage transformation. This phenomenon increased on 90 day of the study, when the presence of collagen and microfibrillar protein material was noticed, and reached its top intensity in 180-day diabetes. Pancreatic fibrosis was also manifested by dilation of walls in pancreatic ducts and vessels, with accompanying fibrocytes present in perivascular spaces. Further intensification of pancreatic excretory ducts and vascular dilation was registered on day 90 of the disease. At this stage eosinophilic granulocytes were observed in the perivascular spaces. Pancreatic fibrosis was the most evident in samples of rabbits with 180-day diabetes. Bundles of collagen fibbers were present both in extra cellular spaces and around vessels and excretory ducts $(5,24)$.

Initial process of glycogen storage in exocrine cells in ultrastructural level was already found in pancreatic preparations of animals with 21 days diabetes. Glycogen began to concentrate around nuclei of exocrine cells. Intensification of this process was proportional to the course of the experiment, and was probably one of the reasons for cellular damage. Regions of deceased cytoplasm occurred in exocrine cells on day 42 of the diabetes. Initial changes in mitochondria, such as increased parenchyma transparency and crests' fragmentation, were the signs of accompanying parenchyma 
degeneration in exocrine cells. It was intensified in the following experimental groups reaching the image of myelin degeneration in 90 days diabetes group. The above observations are consistent with available publications in this subject, and may act as an explanation for the decline of metabolism in pancreatic exocrine cells in the course of diabetes mellitus $(6,7)$. Such an intense destruction in the exocrine cells at the state of absolute insulin deficiency may be a proof of stimulating or even maintaining influence of this hormone for physiological processes in their cytoplasm (11).

It seems, that impaired exocrine pancreatic function in diabetes might be a result of the following factors: 1) glucagon inhibiting influence and lack of insulin stimulation in processes of synthesis in exocrine cells, and 2) while examining alloxan-induced model of diabetes, direct inhibiting influence of this substance on adenylate cyclase should be considered, which through decrease in cAMP level impairs extrareceptor mechanisms in the exocrine cells area. In insulin-dependent diabetes mellitus, both morphology and exocrine pancreatic function appear to be altered, although in humans this phenomenon and the mechanisms responsible for it have not been elucidated in detail (9)

We concluded that the intensification of changes in the ultrastructure of the exocrine part of the pancreas correlated with the changes of the total pancreatic lysosomal lipase activity, and glucose and lipoprotein levels.

\section{REFERENCES}

1. Anderson, L. C., Garrett, J. R., Suleiman, A. H., Proctor, G. B., Chan, K. M., Hartley, R.: In vivo secretory responses of submandibular glands in streptozotocin-diabetes rats to sympathetic and parasympathetic nerve stimulation. Cell. Tissue Res. 274, 559-566 (1993).

2. Assmann, G. (1979): Lipiddiagnostik heute. In: Lipoproteine und Herzinfarkt. Ed: Greten, H., Witzstock-Verlag, Baden-Baden.

3. Aughsteen, A. A.: Morphometric studies on the juxta-insular and tele-insular acinar cells of the pancreas in normal and streptozotocin-induced diabetic rats. J. Electron. Microsc. 42, 79-87 (1993).

4. Barrett, A. J. (1972): Lysosomal enzymes. In Lysosomes. Ed.: Dingle J., North-Holland Publ., Amsterdam.

5. Belfiore, F., Ianello, S., Campione, R., Bombaci, M., Gulisano, Y. (1990): Selected aspects of capillary morphology and metabolism in diabetes. In: Endothelial cell function in diabetic microangiopathy. Eds: Molinatti, G. M., Bar, R. S., Belfiore, F., Porta, M. Problems in methodology and clinical aspects. Karger, Basel.

6. Foulis, A. K., Stewart, J. A.: The pancreas in recent-onset Type 1 (insulin-dependent) diabetes mellitus: insulin content of islets, insulitis and associated changes in the exocrine acinar tissue. Diabetologia 26, 456-461 (1984).

7. Frier, B. M., Saunders, J. H. B., Wormsley, K. G., Bouchier, I. A. D.: Exocrine pancreatic function in juvenile-onset diabetes mellitus. Gut 17, 685-691 (1976).

8. Glauret, A. M.: Practical methods in electron microscopy. Northholand Comp., Amsterdam and Oxford (1974). 
9. Groger, G., Layer, P.: Exocrine pancreatic function in diabetes mellitus. Eur. J. Gastroenterol. Hepatol. 7, 740-746 (1995).

10. Hayat, M. A.: Principles and techniques of electron microscopy. Van Nostrand Reinhold Comp., New York (1970).

11. Khamdan, R., Shubnikova, E. A., Pogodina, L. S.: Rhythmic changes in protein synthesis in mouse pancreatocytes after food stimulation, in alloxan diabetes and in isoproterenol exposure. Biull. Eksp. Biol. Med. 111, 142-144 (1991).

12. Kohlmeier, M.: Direct enzymic measurement of glycerides in cells and in lipoprotein fractions. Clin. Chem. 32, 63-66 (1986).

13. Kuijpers, G. A. J., Van Nooy, I. G. P., De Pont, J. J., Bonting, S. L.: The mechanism of fluid secretion in the rabbit pancreas studied by means of various inhibitors. Biochim. Biophys. Acta 778, 269-276 (1984).

14. Kurahashi, M., Inomata, K.: Amylase secretion by parotid glands and pancreas of diabetic rats during feeding. Am. J. Physiol. 254, 878-882 (1988).

15. Kuschner, B., Lazar, M., Furman, M., Lieberman, T. W., Leopold, I. H.: Resistance of rabbits and guinea pigs to the diabetogenic effect of streptozotocin. Diabetes 18, 542-544 (1969).

16. Lowry, O. H., Rosebrough, N. J., Farr, A. L., Randall, R. J.: Protein measurement with the Folin phenol reagent. J. Biol. Chem. 193, 265-275 (1951).

17. Maciejewski, R., Hermanowicz-Dryka, T., Czerny, K., Wójtowicz, Z., Dryka, T.: Changes of the ATPase, acid phosphatase and alkaline phosphatase reaction intensity in the parotid and submandibular glands of rabbits in experimental diabetes. Folia Histochem. Cytobiol. 37, 99-100 (1999).

18. Maciejewski, R., Burdan, F., Hermanowicz-Dryka, T., Wójcik, K., Wójtowicz, Z.: Changes in the activity of some lysosomal enzymes and in the fine structure of submandibular gland due to experimental diabetes. Acta Physiol. Hung. 86, 127-137 (1999).

19. Novikoff, A. B. (1963): Lysosomes in the physiology and pathology of cells. Contributions of staining methods. In: Ciba Foundation Symposium on Lysosomes. Eds: de Reuck, A. V. S., Churchill, J. A. Little Brown, Boston.

20. Okabayashi, Y., Otsuki, M., Ohki, A., Suehiro, I., Baba, S.: Effect of diabetes mellitus on pancreatic exocrine secretion from isolated perfused pancreas in rats. Dig. Dis. Sci. 33, 711-717 (1988).

21. Oliver, C., Yuasa, Y.: Distribution of basal lysosomes in exocrine acinar cells. J. Histochem. Cytochem. 35, 565-570 (1987).

22. Sato, M., Yamamoto, K., Mayama, H., Yamashiro, Y.: Exocrine pancreatic function in diabetic children. J. Pediatr. Gastr. Nutr. 3, 415-420 (1984).

23. Siedel, J., Hagele, E. O., Ziegenhorn, J., Wahlefeld, A. W.: Reagent for the enzymatic determination of serum total cholesterol with improved lipolytic efficiency. Clin. Chem. 29, 1075-1080 (1983).

24. Siperstein, M. D.: Diabetic microangiopathy and the control of blood glucose. New Engl. J. Med. 309, 1577-1581 (1983). 